\title{
Human Recognition-Behavioral Adaptation System
}

\section{Yutaka Masuda ${ }^{1}$}

Received: 27 April 2020 /Revised: 20 July 2020 / Accepted: 27 July 2020/

Published online: 13 August 2020

(C) The Author(s) 2020

\begin{abstract}
I understand that phenomena are compatibly explained via synthetic reasoning-model inducted from the premise. This is my fundamental researching strategy. Now, some neuroscientists suggested that mammalian brains work via network of the neuronal modules, and I and my colleagues reported that the modules are followed by individual humoral stress-coping glycolipids. Here, I noticed that the module-network followed by the glycolipids could be symbolically structured as a model qualitative- and quantitative-reasoning mammalian recognitionbehavioral stress-coping system. On the other hand, Evolutionary psychologists understand that human recognition-behaviors originate from animal recognition-behaviors, and philologists premise that human operates language and symbols semantically. I symbolized human recognition-behavioral stress-coping system as the self by using a mathematical logic Complex-network, developed the self to the world by using a mathematical logic Topology, and considered the self and the world as synthetic reasoning-model of human recognitionbehavioral adaptation system. Furthermore, I explained human psychological realities: personality, neurotic symptoms, sympathy and empathy, social selection pressure, and nature of psychotherapy via the synthetic reasoning-model. I also analyzed psychotherapeutic strategies of the main psychological parties for clarifying the comprehensions for human recognitionbehavioral adaptation system, and finally, I concluded that my fundamental researching strategy is valid for totally comprehending human adaptation recognition-behaviors.
\end{abstract}

Keywords Human recognition-behavioral adaptation system · Psychological realities · Symbolizing $\cdot$ Synthetic reasoning-model

\section{Introduction}

I understand that phenomena can be fully explained only via a compatible model inducted by qualitative- and quantitative-reasoning from the premise. This is my fundamental researching

Yutaka Masuda

y-masuda@hos.akita-u.ac.jp

1 Psychosomatic Division, Graduate School of Medicine, Akita University, Hondo 1-1-1,

Akita 010-8543, Japan 
strategy, and the points are induction of synthetic reasoning-model from the premise and deduction of phenomena via the model.

Now, some neuroscientists previously suggested that mammalian brains work via the neuronal module-network (Bullmore and Sporn 2012; Perona et al. 2008; Salnyeri and Kovaces 1994), and I and my colleagues reported that the neuronal modules are followed via individual humoral stress-coping glycolipids (Masuda et al. 2019). I noticed that the former is qualitative-reasoning mammalian recognition-behavioral stress-coping system, and the latter is quantitative-reasoning the system. On the other hand, Evolutionary psychology understands that human recognition-behaviors originate from mammalian recognition-behaviors, and philologists premise that human operates language and symbols semantically.

Here, I hypothesized that human recognition-behavioral stress-coping system could be symbolically structured as a synthetic reasoning-model, and that the model could develop for explaining psychological realities belonging to human recognition-behavioral adaptation system.

\section{The Synthetic Reasoning-Model}

\section{The Self}

It was reported that human stress-coping recognition-behaviors are also regulated via network of serotonergic module keeping physical strength by regulating the emotional behaviors, adrenergic module inducing stress-coping behaviors, cholinergic module keeping the stresscoping memories, and dopaminergic module integrating these module-functions with compatibility, and that the modules are followed by individual humoral stress-coping glycolipids (Masuda 2020).

Here, the serotonergic module is defined to $E$ (Emotion)-node, the adrenergic module is to $W$ (Will)-node, the cholinergic module is to $M$ (Memory)-node, and the dopaminergic module is to I (Integration)-node. Sizes of the nodes are corresponding to the activities of the nodes. Inode places in centroid of (M-E-W) triangle because of the integration role. The functional node-network is enclosed with Self-membrane. This is structure of the self. When the self recognizes a strong stress, activities of $M-, E-$, and $W$-nodes change for coping the stress, and the triangle-shape is deformed. The deformation moves I-node from the triangle-centroid, and I-node controls activities of $M-, E$-, and $W$-nodes for putting itself back to the centroid. This is mechanism of compatible adaptation followed by a mathematical logic Complex-network. MW-I circuit induces conditionings. W-E-I circuit induces instinctive behaviors. E-M-I circuit induces emotional behaviors. I-(M-E-W) circuit induces voluntary learning to gain the adaptation. These are functions of the self. Symbolical scheme of the self is showed in (Fig. 1).

I-node recognizes the successful maintenance of the compatibility as pleasure, and does the failed maintenance of the compatibility as uneasy. The self suggests personality: an individual recognition-behavioral adaptation pattern.

\section{The World}

\section{The Basic World}

The self of human, who can use language and symbol semantically, develops to the basic world followed by a mathematical logic Topology. 


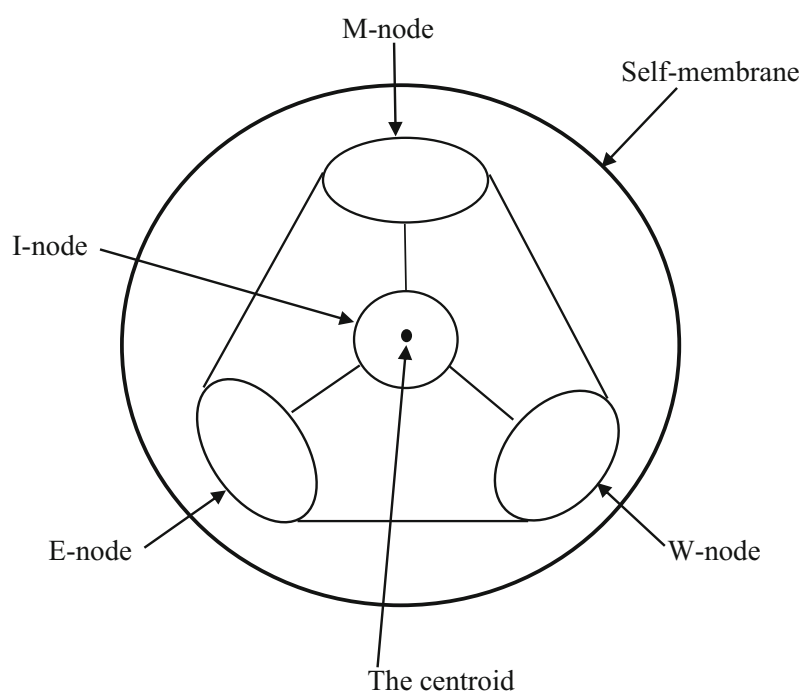

Fig. 1 The self. The self is inducted from mammalian recognition-behavioral stress-coping system which is network of serotonergic module keeping physical strength by regulating emotional behaviors, adrenergic module inducing stress-coping behaviors, cholinergic module keeping stress-coping memories, and dopaminergic module integrating these node-functions with compatibility. The cholinergic module is defined to $M$ (Memory)-node, the adrenergic module is to $W(W i l l)$-node, the serotonergic module is to E (Emotion)-node, and the dopaminergic module is to I (Integration)-node. I-node places at the centroid of (M-E-W) tringle because of role maintaining the compatibility. Self-membrane covers and protects I-(M-E-W). M-W-I circuit induces conditionings. W-E-I circuit induces instinctive behaviors. E-M-I circuit induces emotional behaviors. I-(M-E-W) circuit induces voluntary learning to gain successful stress-copings.

Centroid of the self extends to Bird-eyes view point, and the extension-line is distinguished as Adaptation-axis built-in learning-elements conditionings, pattern-recognition, and modelsimulation. The basic world is filled with linguistic and symbolic association-network, and differs to 3layers-hierarchy. The lower layer is corresponding to instinctive physical adaptation space. The middle layer is corresponding to social mental adaptation space. The upper layer is corresponding to symbolical spiritual adaptation space. The hierarchy is covered by World-membrane which indicates cognition-limit of human. This is structure of the basic world. I-(M-E-W) is rotating along Adaptation-axis via experiences, and is raising forward Bird-eyes view point via learning. This is mechanism of the self-evolution. The self-evolution is significance of existence of the basic world. Symbolical scheme of the basic world is showed in Fig. 2 (explained in the upper legend).

History of the real adaptation is considered that of the self-evolution. The basic world is prescribed by culture: linguistic and symbolic circumstance which humans collectively create and maintain.

\section{The Thinking-World}

The basic world transforms to the thinking-world for maintaining semantic propriety of thinking.

Adaptation-axis is changed to Inference-axis built-in thinking-elements algorism, analogy, and pattern-recognition. Hub-keywords of the association-network are on Inference-axis. Premise is recognized as a set at Basic border by using instinctive discretion and comparison 


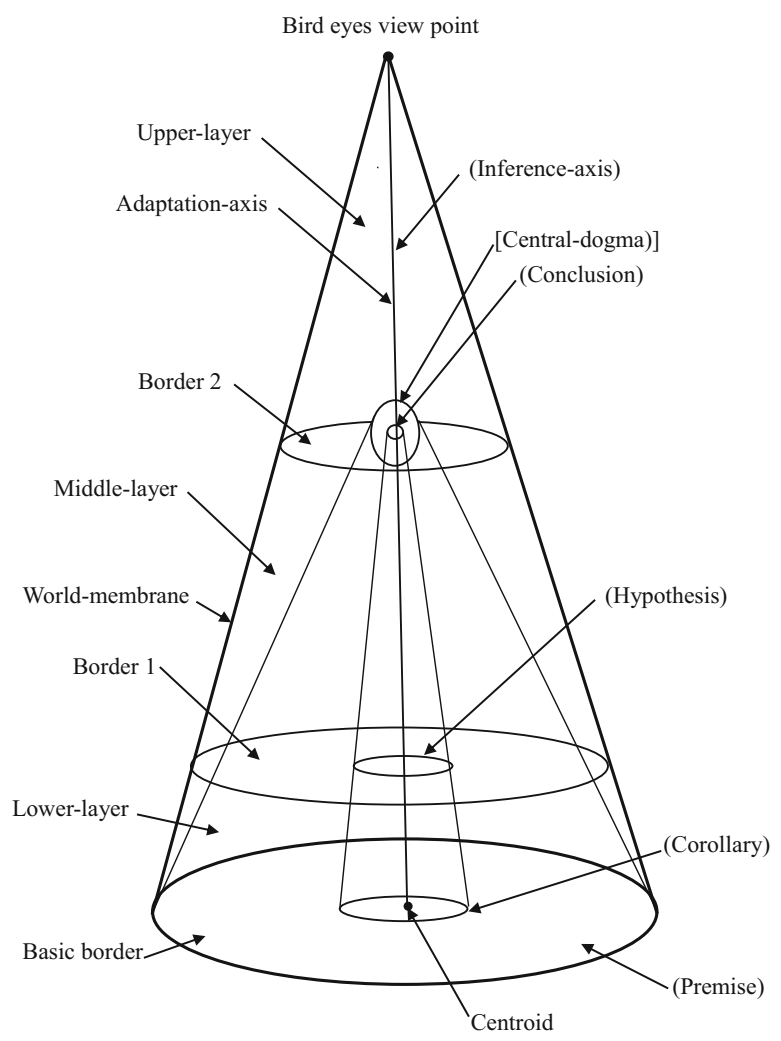

Fig. 2 The world. 1. The basic world. The self is developed to the basic world via topological transformation. The centroid in Basic border expends to Bird-eyes view point, and the expanding line is named to Adaptationaxis built-in learning-elements conditionings, model-simulation and pattern-recognition. The system is differentiated to 3layers-hierarchy. Lower-layer is the instinctive physical space, Middle-layer is the social mental space, and Upper-layer is the symbolic spiritual space. Borders 1 and 2 separate the layers. The self-evolution is induced by rotating and raising of I-(M-E-W) along Adaptation-axis via successful experiences and learning. World-membrane indicates cognition-limit of human. 2. (The thinking-world) The basic world transforms to the thinking-world for maintaining semantic property of thinking. Adaptation-axis changes to Inference-axis built-in the thinking elements algorism, analogy, and pattern-recognition. Premise is set around the centroid, and Corollary which is separated from the premise, is mapped as Hypothesis to Border 1. The hypothesis is mapped as Conclusion to Border 2. This upward of I-(M-E-W) along Inference-axis is called as induction. Downward of I-(M-E-W) along Inference-axis is called as deduction followed by a mathematical logic. 3. [Central-dogma] In the thinking-world, I-node gaining "corollaries-conclusions" develops to Central-dogma. Central-dogma generalizes them as "premise-conclusion" via the thinking-economy. This generalizing manner is called as humanistic. Humanistic induces the human-specific inference-manner abduction. Synthetic reasoning-model is presented as a scientific aspect of Central-dogma.

named to Gestalt. Corollary is distinguished from the premise via pattern-recognition. The corollary is mapped to Border 1, and is described as Hypothesis via analogy using a natural language. The hypothesis is mapped to Border 2, and is described as Conclusion via algorism. This upward of I-(M-E-W) along Inference-axis is called as induction. The downward of I-(ME-W) along Inference-axis is called as deduction and deduction must be followed by a mathematical logic. Symbolic scheme of the thinking-world is showed in Fig. 2 (explained in the middle legend). 
I-node in the thinking-world performs pattern-recognition, analogy, and algorism in this order for one object. This is called as Scientific method.

\section{Central-Dogma}

I-node in the thinking-world also develops to Central-dogma.

Algorism of artificial intelligence can be completely and not-restrictedly traced via the stronger logical engine; however, algorism of I-node might be incomplete and restricted. For compensating for the deficit, human invents abduction which searches a compatible logic retrospectively. Here, Inode which gains corollaries-conclusions via successful learning and experiences has evolved to Central-dogma generalizing the harvests as a simple premise-conclusion via the thinking-economy. Synthetic reasoning-model is presented as scientific aspect of Central-dogma. Symbolical structure of Central-dogma is showed in Fig. 2 (explained in the lower legend).

Generalizing manner of Central-dogma is called as humanistic. Humanistic often induces Cognitive bias (Wikipedia 2019), and the cognitive bias induces Fallacy (Wikipedia 2019). Central-dogma induces Intrusive thought (Wikipedia 2019) for decreasing the uneasiness; however, Central-dogma considers learning and experiences increasing pleasure as Flow (Wikipedia 2019). Flow induces transcendent Tacit knowledge (Wikipedia 2019). Central-dogma also forms the self-image according to humanistic.

\section{Human Psychological Realities Explained via the Model}

\section{Personality}

Performance of functional nodes-network is determined by function of the dominant node, and so, personality is also estimated via the function of the dominant node.

Person having a typical personality shows a strategical recognition-behavioral adaptation pattern corresponding to the dominant node. I-node size is related to Paranoid, Schizoid, or Schizotypal personality, and the strategy is the cognitive compatibility-maintenance. The size is also related to Obsessive-compulsive or Dependent personality, and the strategy is decrease of uneasiness via the stereotypic behaviors. W-node size is related to Borderline personality, and the strategy is aggression to control the human relationship. The node size is also related to Avoidant personality, and the strategy is flight from the struggle. M-node size is related to Narcissistic personality, and the strategy is mimicry to the cultural model. The size is also related to Histerionic personality, and the strategy is recognition-behavioral transformation via dissociation and/or conversion. Persons having typical personalities often show a common adaptation strategy, so-called Co-dependence.

Quality of node-activity is epigenetically determined, and the quality is indicated as vectors. M-node has vectors of memorizing, recollecting, and remembering. I-node has vectors toward M-node, E-node, and W-node, and toward the Bird-eyes view point. As I-node vectors-shapes of humans of Developmental disability are different from those of general humans, humans of Developmental disability are more and less exposed of the social selection pressure. Humans of Intellectual Giftedness who are gifted an extraordinary cognition-ability, are forced to invent the original learning-manners. Both of them often show Over-excitability for maintaining the I-node vectors-centroid. W-node has vectors toward the self and toward the others. The vectortoward-self is observed as flight, and the vector-toward-others is done as fight. E-node also has vectors toward the self and the others. The vectors might be called as love and/or affection. 
Human can phenomenologically describe the self as the self-image. Personality tests have tried to reason the phenomenological descriptions for understanding the self.

\section{Neurotic Symptoms}

Human in stressful situation shows neurotic symptoms. The symptoms are explained via functions of circuits of the self.

Symptoms corresponding to emotion are induced via E-M-I circuit. In the circuit, E-node dysfunction induces fatigue, and I-node dysfunction does anxiety. Symptoms corresponding to conditionings are induced via M-W-I circuit. In the circuit, M-node dysfunction induces obsession and/or compulsion. As I-node requires prompt dopaminergic satisfaction, the human who can easily find objects increasing the prompt dopaminergic satisfaction easily falls into abuse. Symptoms corresponding to instincts are induced via W-E-I circuit. In the circuit, W-node settingon the flight induces dissociation and/or conversion, and W-node setting-on the fight induces aggression. I-node in the anxious state induces self-injuring for getting the prompt dopaminergic satisfaction. When Central-dogma considers itself as unbearable stressor, the human might try committing-suicide for eliminating itself. I-node dysfunction in I-(M-E-W) circuit induces $A d$ justment disorders, and somatization is often induced via the conversion mechanism.

Neurotic patients are generally fatigue and fall into depressive state because of many efforts coping the stresses, and so, the situation-controlling is often therapeutic for them.

\section{Sympathy and Empathy}

It was previously reported that mirror neuron maintains human empathy (Carr et al. 2003; Pfeifer et al. 2008). I premise that mirror neuron is belonging to I-node.

Human in uneasy situation shows emotional behaviors. Another human whose I-node is placing in the instinctive physical layer or in the mental social layer is affected by the emotional behaviors, and shows the same emotional behaviors for dealing with the uneasy situation. This is mechanism of sympathy. Sympathy also induces Peer pressure (Wikipedia 2019). On the other hand, human whose I-node is placing in the symbolic spiritual layer can objectively observe emotional behaviors of the others, and so, the human can surmise origin of the emotional behaviors without inducing the emotional behaviors. This is mechanism of empathy. Namely, human experiences sympathy, and learns empathy.

Humans of Psychopath are considered to lack sympathy toward the others; however, they do not always lose empathy toward themselves.

\section{Demands of the World Layers}

Erikson and Maslow, A. (Wikipedia 2019) individually suggested that Central-dogma should respond to demands of the belonging layer. If Central-dogma does not respond to the demands, it would be exposed by the strong selection-pressure of the layer.

Central-dogma in the physical layer must respond to the physical demands. Central-dogma in the social layer must respond to behavioral standards and roles prepared in the cultural situation for surviving through struggle of the existence in the cultural group. Nevertheless, Central-dogma in the social layer can gain positive approval and estimation by responding to the standards and the roles. The positive approval and estimation induce the cultural adaptation-superiorities honor, finance, and so on, and the superiorities induce pleasure to 
Central-dogma. Furthermore, Central-dogma in the social layer can also find out models having much more adaptation-ability and rivals interrupting to gain positive estimation, by comparing with the self-image. Originally, the spiritual layer is specialized for escaping from the social layer severe to live. Central-dogma in the spiritual layer can obtain the absolute positive approval and estimation even by responding to demands of the self-image. Maslow suggested that human whose Central-dogma is in the spiritual layer, often looks to say arrogantly and to act outrageously.

\section{Nature of Psychotherapy}

Chomsky suggested that human has an inheritance semantically-using language and symbols.

Language and symbols look like to take on attributes of the selfish gene. New connotation inspired into an association-node transforms the association-network for keeping the semantical consistency. This is called to the spirit of words. The spirit of words which is gained via successful learning and experiences induces awareness accelerating the self-evolution, and the self-evolution would dissolve the present psychological dis-adaptation. Psychotherapy is a method accelerating self-evolution of the clients by utilizing the spirit of words. Actually, psychotherapists understand history of self-evolution of the clients, identify causes of their dis-adaptation with empathy, and accelerate their self-evolution by giving positive approval and estimation via successful learning and experiences. Successful learning and experiences of the physical layer are followed by conditionings, those of the mental layer are followed by pattern-recognition and model-simulation, and those of the spiritual layer are followed via transcendent tranquility.

As psychotherapy is a kind of persuasion, psychotherapy is valid only when the client has a motivation for the self-evolution. Furthermore, clients who do not understand nature of psychotherapy might consider psychotherapy as a magical method.

\section{Psychotherapeutic Strategies of Main Psychological Parties}

\section{The Significance}

I consider that psychotherapeutic strategies of main psychological parties are expressing comprehensions of the parties for human recognition-behavioral adaptation system. Here, I analyze the psychotherapeutic strategies by using the synthetic reasoning-model.

\section{Cognitive-Behavioral Science}

Cognitive-Behavioral Science has developed based upon investigations of mammalian conditionings.

Behavioral Therapy mainly deals with human conditionings in the physical layer. Cognitive Therapy deals with the successful conditionings in the social layer. Cognitive destruction tries to delete the rigid conditionings inducing invalid adaptation-behaviors. Social Skill Training is a social operant-conditioning method for the clients to increase positive self-approval and self-estimation.

\section{Psychoanalysis}

Psychoanalysis is interested in dynamism of human mentality, and mainly analyzes patternrecognition and model-simulation of Central-dogma in the social layer. 
Psychoanalysts premise sufficient I-node ability of the client, so, they have made the importance on language and symbols. The founder Freud, S. (Wikipedia 2019) noticed common structure of the self-image, and tried to clarify the structure by listening to the clients-narratives. Adler emphasized social hierarchy, and advocated sense of inferiority in Individual Psychology. Erikson investigated Developmental task in the social world. Jung found Archetypes beyond the cultures. And Reich adhered Orgone: Flow-effect of sexual intercourse. Open dialogue tries to give social positive approval and estimation to schizophrenic patients by listening their narratives closely. Family therapy considers family as a cultural system, and points out double-binging forcing dis-adaptation to the client. Schema Therapy suggests that the self-image of the client is formed by cultural roles in the belonging society.

\section{Humanistic Psychology}

Humanistic Psychology positively estimates humanistic of Central-dogma. The party has tried to integrate Cognitive-Behavioral Science and Psychoanalysis.

Psychotherapists belonging to this party recommend for the clients to take the self-images as they are. Supporting Psychotherapy premises insufficient I-node ability of the client, and tries to change the stressful collective situation to the stress-less one. Group therapy tries to increase co-estimation among the clients by utilizing physical arts and/or sports.

\section{Strategy in the Spiritual Layer}

The spiritual layer is filled with the individual successful association-network, and always gives absolute acceptance and estimation to Central-dogma. Central-dogma in the layer can perform an original discovery and a magical invention under the transcendent tranquility; nevertheless, the discovery and the invention are corresponding to quality and quantity of the association-network.

I think that Psychoanalysis and Humanistic psychology did not prepare psychotherapeutic strategies in the spiritual layer. However, pioneers of Cognitive-Behavioral Science accepting ideas of Humanistic psychology discovered Meditation which induces a transcendent tranquility the same as Flow does. I think that Meditation is handled as a main method of Mindfulness and Japanese Naikan therapy in the present time.

When Central-dogma reaches to Bird-eyes view point via the transcendent tranquility, the soul (self-image) unites to the body (physical-adaptation-standard) entirely. This is mechanism of identification. Kubler-Ross suggested that the identification would enable to transcend even the death.

\section{General Discussion}

I am very interested in human recognition-behavioral adaptation system, and so, I have investigated the system basically and clinically.

Frankly speaking, I was not satisfied with comprehensions of the main psychological parties for the system. Psychoanalysis founder Freud schemed human mental structure symbolically, but I think that he only tried to qualitative-reason the self without any quantitative-reasoning procedures. Maslow, who presented idea of Humanistic psychology, 
also schemed human mental hierarchy symbolically, but I think that he only qualitativereasoned the basic world. Cognitive-Behavioral Science has successfully explained human adaptation recognition-behaviors; however, I am afraid that the psychological party might not enough qualitative-reason the world. Therefore, for integrating these unbalanced comprehensions, I inducted the synthetic reasoning-model from the premise of human recognitionbehavioral adaptation system, and explained human psychological realities via the model.

Finally, I conclude that my fundamental researching strategy is also valid for totally comprehending human recognition-behavioral adaptation system.

Acknowledgments I utilize PubMed for getting basic and clinical medical literatures. I cited the presented Specific psychological term or Key-person of clinical psychology from Wikipedia, because I could get them via PubMed.

Author Contribution I prepared all of the manuscript.

Availability of Data and Material Not applicable.

\section{Compliance with Ethical Standards}

Conflict of Interest The author declares that he has no conflict of interest.

Ethics Approval Not applicable.

Code Availability Not applicable.

Consent to Participate Not applicable.

Consent for Publication Not applicable.

Open Access This article is licensed under a Creative Commons Attribution 4.0 International License, which permits use, sharing, adaptation, distribution and reproduction in any medium or format, as long as you give appropriate credit to the original author(s) and the source, provide a link to the Creative Commons licence, and indicate if changes were made. The images or other third party material in this article are included in the article's Creative Commons licence, unless indicated otherwise in a credit line to the material. If material is not included in the article's Creative Commons licence and your intended use is not permitted by statutory regulation or exceeds the permitted use, you will need to obtain permission directly from the copyright holder. To view a copy of this licence, visit http://creativecommons.org/licenses/by/4.0/.

\section{References}

Bullmore, E., \& Sporn, O. (2012). The economy of brain network organization. Nature Reviews. Neuroscience, 13, 336-349. https://doi.org/10.1038/nrn3214.

Carr, L., Iacoboni, M., Dubeau, M. C., Mazziotta, J. C., \& Lenzi, G. L. (2003). Neural mechanisms of empathy in humans: A relay from neuronal systems for imitation to limbic areas. Proceedings of the National Academy of Sciences of the United States of America, 100, 5497-5502. https://doi.org/10.1073/pnas.o935845100.

Cognitive bias. Wikipedia. (2019). https://en.wikipedia.org/wiki/Cognitive-bias

Fallacy. Wikipedia. (2019). https://en.wikipedia.org/wiki/Fallacy

Flow. Wikipedia. (2019). https://en.wikipedia.org/wiki/Flow-(psychology).

Freud, S. Wikipedia. (2019). https://en.wikipedia.org/wiki/sigmund-Freud 
Intrusive thought. Wikipedia. (2019). https://en.wikipedia.org/wiki/Intrusive-thought

Maslow, A. Wikipedia. 2019, https://en.wikipedia.org/wiki/Abraham-Maslow

Masuda, Y. (2020). A humoral recognition-behavioral stress-coping glycolipid considered as another biomarker of psychotic symptoms of schizophrenia. Advances in Neurology and Neuroscience., 2020, 3, 37-40 https://www.opastonline.com/previous-issue-an/AN.03.01.07.

Masuda, Y., Narita, H., \& Hasegawa, H. (2019). Stress-coping humoral glycolipids produced by mice given controlled bathing treatments. Hindawi Neuroscience Journal, 2019. https://doi.org/10.1155/2019/4972186.

Peer pressure. Wikipedia. (2019). https://en.wikipedia.org/wiki/Peer-pressure

Perona, M. T., Waters, S., Hall, F. S., Sora, I., Lesch, K. P., Murphy, D. L., et al. (2008). Animal model of depression in dopamine, serotonin, and norepinephrine transporter knockout mice: Prominent effects of dopamine transporter deletions. Behavioural Pharmacology, 19, 566-574. https://doi.org/10.1097/FBP.0b0 Be32830cd80f.

Pfeifer, J. H., Iacoboni, M., Mazziotta, J. C., \& Dapretto, M. (2008). Mirroring others' emotions relates to empathy and interpersonal competence in children. Neuroimage., 39. https://doi.org/10.1371/journal. pbio.0030079.

Salnyeri, Z., \& Kovaces, G. L. (1994). Role of oxytocin in neuroadapation to drugs of abuse. Psychoneuroendocrinology., 19, 85-117.

Tacit knowledge. Wikipedia. (2019). https://en.wikipedia.org/wiki/Tacit-knowledge

Publisher's Note Springer Nature remains neutral with regard to jurisdictional claims in published maps and institutional affiliations. 